\title{
Effects of Changing Guidelines on Prescribing Aspirin for Primary Prevention of Cardiovascular Events
}

\author{
Jennifer Hissett, MD, Brittany Folks, MD, Letoynia Coombs, MS, \\ William LeBlanc, PhD, and Wilson D. Pace, MD
}

Objective: The use of low-dose aspirin for primary prevention of cardiovascular events in patients at elevated risk for cardiovascular disease (CVD) is increasingly being questioned. Aspirin may not benefit this population and may increase the risk of major bleeding events. Data support aspirin use in patients with known CVD.

Methods: This is a secondary analysis of de-identified electronic health record (EHR) data from 131,050 individuals with known CVD or elevated risk for CVD as determined by diagnostic, demographic, and clinical data collected from 33 primary care practices in 11 different clinical organizations across 6 states. The percentage of the population of each cohort with aspirin recorded on their medication list, created through risk base analysis, was observed across 4 time periods.

Results: From 2007 to 2011, aspirin usage reflected in the EHR increased for the entire population and for each individual high-risk diagnosis. The percentage of the population initiating aspirin therapy for primary prevention within a year of diagnosis of CVD risk factors or CVD "equivalency" increased between 2007 and 2011. Among those with a new diagnosis of CVD, aspirin usage also steadily increased over the 4-year period, indicating no negative impact from new negative primary prevention studies.

Conclusions: Primary care clinicians have a central role in providing evidence-based preventive services and should integrate revised information into their practice to improve outcomes. Even with new evidence against the use of aspirin for primary prevention, it is difficult to change beliefs about the effectiveness and safety of aspirin, as reflected in the behavior of physicians and patients. $(\mathrm{J}$ Am Board Fam Med 2014;27:78-86.)

Keywords: Aspirin, Cardiovascular Diseases, Primary Prevention

In 2002 the US Preventive Services Task Force began recommending low-dose aspirin as a primary prevention measure in patients at high risk of developing cardiovascular disease (CVD), defined as acute coronary syndrome or thrombotic cerebral vascular disease, when the benefit outweighs the risk. ${ }^{1,2}$ This idea is increasingly being challenged.

This article was externally peer reviewed.

Submitted 14 January 2013; revised 24 July 2013; accepted 5 August 2013.

From the Louisiana State University Emergency Medicine Residency, New Orleans (JH); the Exempla St. Joseph Hospital Internal Medicine Residency, Denver, CO (BF); and the Department of Family Medicine, University of Colorado School of Medicine, Aurora (LC, WL, WDP).

Funding: Funding provided by the National Heart, Lung, and Blood Institute Grant 5RC1HL101071-02 (Karl Hammermeister, primary investigator).
There is strong evidence that aspirin is beneficial for secondary prevention (prevention of overt symptoms or signs after the disease process has begun) of cardiovascular events. ${ }^{3-5}$ However, no studies have shown the benefits of aspirin to outweigh the risks for those without CVD. ${ }^{6-9}$ The US Food and Drug Administration has twice denied requests to approve aspirin for the primary preven-

Prior presentation: Preliminary findings were presented at the Annual Meeting of the North American Primary Care Research Group, Banff, Alberta, Canada, November 12-16, 2011.

Conflict of interest: none declared.

Corresponding author: Wilson D. Pace, MD, University of Colorado Department of Family Medicine, Mail Stop F496, 12631 E. 17th Ave, Aurora, CO 80045 (E-mail: Wilson. pace@ucdenver.edu). 
tion of cardiovascular events (which include both cardiac and cerebral events) in any population because of a lack of evidence supporting its efficacy. ${ }^{10}$ Furthermore, research in patients with CVD risk equivalents (diabetes, chronic kidney disease, peripheral vascular disease) has demonstrated that the benefit of low-dose aspirin does not outweigh the harm. ${ }^{11-15}$

Multiple studies and meta-analyses indicate that there is no specific population in which the benefits of low-dose aspirin therapy for primary prevention exceed the risks. The Dialysis Outcomes and Practice Patterns Study (DOPPS), an observational investigation of aspirin use and cardiovascular morbidity and mortality among 28,320 patients receiving hemodialysis, found that among all patients with chronic kidney disease both with and without CVD, aspirin was associated with a decreased risk of thrombotic stroke but an increased risk of myocardial infarction and cardiac events. ${ }^{11}$ The inclusion of patients with known CVD make a positive finding of stroke difficult to interpret.

The Prevention of Progression of Arterial Disease and Diabetes (POPADAD) trial examined the effects of aspirin and antioxidants, alone or in combination, among 1276 patients $>40$ years old with diabetes and asymptomatic peripheral artery disease in the absence of CVD. After 8 years of followup, the investigators found no reduction in the incidence of myocardial infarction, stroke, or deaths from CVD. ${ }^{12}$ In the Japanese Primary Prevention of Atherosclerosis with Aspirin for Diabetes (JPAD) trial, 2539 patients with type 2 diabetes were followed for 4 years. Low-dose aspirin did not reduce the risk of cardiovascular events and may have contributed to major gastrointestinal bleeds. ${ }^{13}$

In 2009, the Antithrombotic Trialists' Collaboration (ATTC) published a meta-analysis of 6 primary prevention trials (including 95,000 subjects with low overall average risk) and 16 secondary prevention trials (including 17,000 subjects with high overall average risk) comparing aspirin use with control. While they noted a decrease in nonfatal myocardial infarction among those taking aspirin for primary prevention, they also observed an increase in major bleeding events (both intracranial and gastrointestinal). They concluded that the risk of bleeding among those taking aspirin for primary prevention was increased by risk factors for coronary disease, and therefore guidelines recommend- ing aspirin use for anyone with moderate to high risk of CVD should be reconsidered. ${ }^{14}$

A 2010 trial, Aspirin for Asymptomatic Atherosclerosis (AAA), addressed whether those without CVD but with a low Ankle-Brachial Index score would benefit from low-dose aspirin therapy. The investigators studied 3350 subjects with a low AnkleBrachial Index score in the absence of CVD who were randomly assigned to receive either entericcoated aspirin or placebo and followed for 8 years. There was no significant difference in thrombotic vascular events between the 2 groups. However, investigators found that the risk of a major bleeding event requiring hospitalization was higher among those taking aspirin. ${ }^{15}$

In 2010, the American Diabetes Association (ADA) and the American Heart Association (AHA)/ American College of Cardiology changed their guidelines concerning the use of aspirin for primary prevention. The ADA previously recommended daily low-dose aspirin for all patients older than age 40 , with diabetes, and with at least one other risk factor, such as hypertension. ${ }^{16-18}$ The ADA recommendation now asserts that low-dose aspirin (75-162 mg/day) be considered for primary prevention of CVD for patients with diabetes with a $>10 \%$ 10-year risk of CVD when the benefits outweigh the risks. This would include men $>50$ and women $>60$ years old who have diabetes and one other risk factor such as hypertension, smoking, dyslipidemia, or proteinuria. But, as outlined above, studies have not elucidated a single population in which the benefits of primary prevention exceed the risks. ${ }^{19}$

Primary care physicians (PCPs) have a central role in providing evidence-based preventive services and should incorporate revised information into their practice to improve patient outcomes. However, it is unknown how quickly PCPs are accepting new evidence about the lack of benefit of aspirin for primary prevention. Given the inclusion of the use of aspirin for primary prevention in national quality metrics and various pay-for-performance programs, it may be difficult to change the behavior of PCPs and patients. We initiated an investigation to determine whether recently published studies, ${ }^{11-13,15}$ meta-analyses, ${ }^{14}$ and changing guidelines ${ }^{2,16-18}$ had an effect on aspirin prescribing practices of PCPs who are members of a national electronic practice-based research net- 
work, the Electronic Quality Improvement and Research Network (eNQUIRENet).

\section{Methods}

To observe how recorded aspirin use changed over time, we used an eNQUIRENet database originally created as part of a CVD learning community grant. ${ }^{20}$ The original limited dataset included information on all individuals $>17$ years old from 33 primary care practices in 11 different clinical organizations spread across 6 states. This dataset was fully de-identified by converting dates of service to date ranges corresponding to the dates of the availability of new information or guideline changes related to aspirin for the primary prevention of CVD. From this de-identified data set, information on 131,050 patients was abstracted to create cohorts of patients who met various criteria for primary prevention.

The de-identified dataset included $\geq 4$ years of data for the following information extracted from electronic health records (EHRs): diagnoses (rare diagnoses suppressed) with initial date and follow-up dates recorded in the EHR, medications recorded in the EHR, year of birth (suppressed to 95 years if $>89$ years of age), blood pressure, weight, height, smoking status, total cholesterol, high-density lipoprotein cholesterol, low-density lipoprotein cholesterol, hemoglobin A1c, creatinine, and urine protein-to-creatinine ratio.

From the dataset described above, a retrospective cohort study was conducted comparing (1) patients with a known diagnosis of CVD and (2) patients without CVD but with increased risk of CAD. Patients were identified as having a $>10 \%$ 10 -year risk of CVD using the Framingham scoring system ${ }^{21}$ based on age, sex, blood pressure, cholesterol, smoking status, and a diagnosis of diabetes. Cohorts were conservatively developed to ensure a calculated Framingham 10-year CVD risk score of $>10 \%$ for the entire cohort. For instance, men older than 50 with diabetes and one other risk factor, such as hypertension or hyperlipidemia, all have a Framingham 10-year risk score of $>10 \%$; therefore, this cutoff was used (full cohort definitions for each group are available from the authors). Information used to create the cohorts included diagnoses, average blood pressures (last 3), average non-high-density lipoprotein total cholesterol (last 3 measurements), smoking status, age, and sex.
The full cohort also was organized into subgroups by diagnosis (diabetes, chronic kidney disease, peripheral vascular disease and aortic aneurysm, hyperlipidemia, and hypertension) for presentation and to observe any variance by underlying diagnoses. Changes in recorded aspirin use were observed among patients who had either previous or new diagnoses of CAD risk equivalents within a 4-year period. The periods correspond with the publication of DOPPS in 2007 (time 1: January 1, 2007, to December 31, 2007); POPADAD and JPAD in 2008 (time 2: January 1, 2008, to December 31, 2008); ATTC in 2009 (time 3: January 1, 2009 to May 31, 2010), and the ADA/AHA/American College of Cardiology guideline changes in mid-2010 (time 4: June 1, 2010, to May 31, 2011). Aspirin usage was determined by electronic review of medication lists. Aspirin was identified through review of National Drug Codes (NDCs) from the Medispan drug database ${ }^{22}$ and by reviewing text entries for medications with no associated NDCs. More than $60 \%$ of all aspirin users were identified through NDC matches, and more than $95 \%$ of these were for low-dose aspirin. The text-based aspirin records were grouped and the top 250 records were manually reviewed, representing more than $80 \%$ of all non-NDC-matched aspirin options. Of these, again more than $95 \%$ were for low-dose aspirin. Thus the vast majority of individuals taking aspirin in this study were using it for some type of prevention, not for pain or anti-inflammatory indications.

A total of 131,050 individuals met the criteria for inclusion in one or more of the cohorts for analysis. Individuals could change cohorts from one time period to another based on new diagnoses or clinical data within a given period. Individuals could be in more than one primary prevention subanalysis cohort, but once they received a diagnosis of CVD they remained in this cohort for the remainder of the periods analyzed.

Each cohort by period was broken into 2 groups: met cohort definition before the time period (current) or entered the cohort during the time period (new). This allowed for an analysis of the percentage of patients starting aspirin therapy based on new clinical information over time. For each cohort, the population was determined based on clinical data. Medication lists were subsequently analyzed to determine the percentage of the cohort receiving aspirin therapy. For all known CVD cohorts, clopidogrel was included as an aspirin equiv- 
alent. The study was approved by the Colorado Multi-Institutional Review Board.

\section{Results}

Table 1 summarizes the distribution of the 131,050person cohort by sex, average age, and diagnosis in a hierarchical fashion. The overall subpopulation used in this analysis has slightly more males than females (50.6\% vs $49.4 \%$ ), and the average age of women in each cohort is older than the males, with the exception of the group with peripheral vascular disease.

Table 2 displays the number of individuals with diagnoses of interest (CVD risk equivalency for primary prevention and diagnosed CVD for secondary prevention) by time period and the number of people taking aspirin, and the percentage of each cohort that is taking aspirin. It is further separated by individuals with a diagnosis established before the first day of the period (current) and those individuals who were newly diagnosed during a given time period (new). Given that individuals can be included in more than one primary prevention cohort, the totals per cohort exceed the total population studied. The table demonstrates an increase in the percentage of individuals recorded as using aspirin for primary prevention over time, with the exception of hyperlipidemia and hypertension in 2008. There is no indication of any consistent decrease in aspirin usage over time related to individuals who newly enter the groups with a $>10 \%$ 10 -year Framingham risk score for a future coronary artery event.

Table 3 shows the total number of patients with new or current disease taking aspirin. Figure 1 displays the percentage of the entire study cohort recorded as using aspirin across the various periods by diagnosis. From 2007 to 2011, aspirin usage reflected in the EHR increased for the entire cohort and for each individual diagnosis. Aspirin usage for secondary prevention is displayed in Figure 2. Among those with a new diagnosis of CVD, aspirin usage has steadily increased over the 4-year period, indicating no negative effects from negative primary prevention studies during this time period.

Figure 3 displays the percentage of individuals recorded as using aspirin with a new diagnosis of CVD risk factors or CVD equivalency during each period. In all cases the percentage of the population initiating aspirin therapy within a year of a new diagnosis went up between 2007 and 2011.

\section{Discussion}

Despite evidence disputing the benefit of aspirin for primary prevention of CVD events, as well as changing guidelines, patients in the observed practices demonstrated increased aspirin use for primary prevention across all groups with CVD risk equivalents. The data do not indicate any effect on the use of aspirin for primary prevention as a result of new negative primary prevention trials and minor changes in ADA/AHA guidelines that were published between 2007 and 2010. After the time period evaluated in this project, Sheshasai et $\mathrm{al}^{23}$ published a meta-analysis that includes all 9 randomized controlled trials of aspirin used for primary prevention of CVD, extending previous work through the addition of the latest 3 trials. The analysis included data on $>100,000$ people and $>700,000$ person-years. An approximate 10\% decrease in nonfatal myocardial infarctions was seen, but there was no change in all-cause cardiovascular or cancer death rates. The nontrivial bleed rates exceeded the decrease in nonfatal myocardial in-

Table 1. Distribution of Cohort by Sex, Age (with Standard Deviation [SD]), and Diagnosis

\begin{tabular}{lcccccc}
\hline & & \multicolumn{2}{c}{ Male } & & \multicolumn{2}{c}{ Female } \\
\cline { 3 - 3 } \cline { 5 - 6 } Diagnosis & Total (n) & Patients (n) & Age, years (SD) & & Patients (n) & Age, years (SD) \\
\hline Cardiovascular disease & 13,639 & 7,817 & $68.8(12.2)$ & & 5,816 & $71.4(13.7)$ \\
Diabetes & 25,293 & 12,780 & $59.7(14.3)$ & & 12,509 & $61.3(15.1)$ \\
Chronic kidney disease & 4,304 & 2,351 & $68.2(14.4)$ & & 1,952 & $71.1(15.0)$ \\
Peripheral vascular disease & 4,266 & 1,871 & $67.8(13.6)$ & & 2,395 & $63.6(17.9)$ \\
Aortic aneurysm & 952 & 663 & $71.8(12.1)$ & & 289 & $72.7(14.5)$ \\
Hyperlipidemia & 94,538 & 50,350 & $55.0(13.9)$ & & 44,169 & $59.1(14.6)$ \\
Hypertension & 74,850 & 35,952 & $58.4(14.3)$ & & 38,882 & $62.2(14.7)$ \\
Total & 131,050 & 66,327 & $55.1(14.4)$ & & 64,699 & $58.6(15.2)$ \\
\hline
\end{tabular}




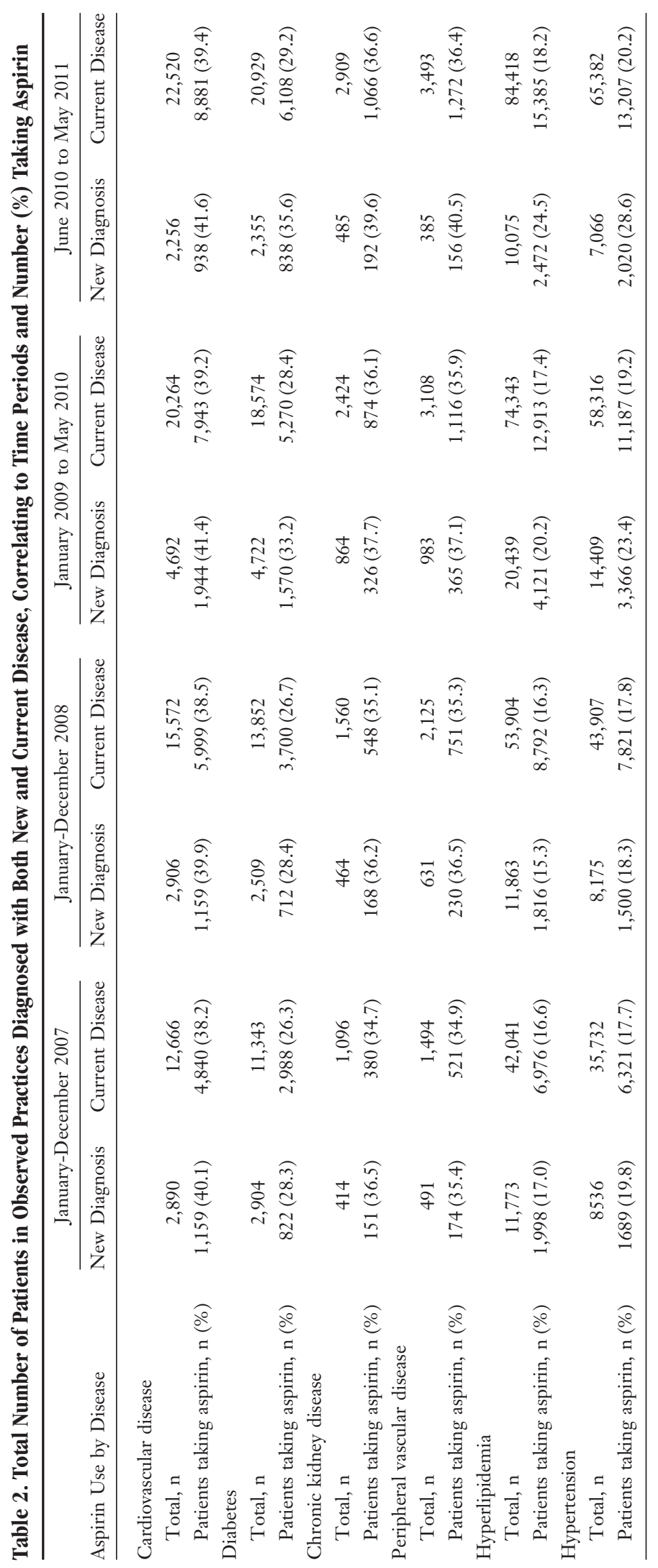


Table 3. Total Number of Patients Diagnosed with Both New and Current Disease and Taking Aspirin Therapy

\begin{tabular}{|c|c|c|c|c|c|c|c|c|}
\hline \multirow[b]{2}{*}{ Disease } & \multicolumn{2}{|c|}{$\begin{array}{l}\text { January-December } \\
2007\end{array}$} & \multicolumn{2}{|c|}{$\begin{array}{l}\text { January-December } \\
2008\end{array}$} & \multicolumn{2}{|c|}{$\begin{array}{c}\text { January } 2009 \text { to } \\
\text { May } 2010\end{array}$} & \multicolumn{2}{|c|}{$\begin{array}{l}\text { June } 2010 \text { to } \\
\text { May } 2011\end{array}$} \\
\hline & $\begin{array}{c}\text { New } \\
\text { Diagnosis }\end{array}$ & $\begin{array}{l}\text { Current } \\
\text { Disease }\end{array}$ & $\begin{array}{c}\text { New } \\
\text { Diagnosis }\end{array}$ & $\begin{array}{l}\text { Current } \\
\text { Disease }\end{array}$ & $\begin{array}{c}\text { New } \\
\text { Diagnosis }\end{array}$ & $\begin{array}{l}\text { Current } \\
\text { Disease }\end{array}$ & $\begin{array}{c}\text { New } \\
\text { Diagnosis }\end{array}$ & $\begin{array}{l}\text { Current } \\
\text { Disease }\end{array}$ \\
\hline Cardiovascular disease & 1,159 & 4,840 & 1,159 & 5,999 & 1,944 & 7,943 & 938 & 8,881 \\
\hline Diabetes & 822 & 2,988 & 712 & 3,700 & 1,570 & 5,270 & 838 & 6,108 \\
\hline Chronic kidney disease & 151 & 380 & 168 & 548 & 326 & 874 & 192 & 1,066 \\
\hline $\begin{array}{l}\text { Peripheral vascular disease } \\
\text { and aortic aneurysm }\end{array}$ & 174 & 521 & 230 & 751 & 365 & 1,116 & 156 & 1,272 \\
\hline Hyperlipidemia & 1,998 & 6,976 & 1,816 & 8,792 & 4,121 & 12,913 & 2,472 & 15,385 \\
\hline Hypertension & 1,689 & 6,321 & 1,500 & 7,821 & 3,366 & 11,187 & 2,020 & 13,207 \\
\hline
\end{tabular}

farctions. The positive results related to nonfatal myocardial infarctions were heavily influenced by older studies, which were conducted before more aggressive treatment of hypertension and hyperlipidemia. The authors conclude that "routine use of aspirin for primary prevention is not warranted."

The data in this study indicate that there has been no decrease in the clearly appropriate use of aspirin in individuals with known CVD. It is reassuring that negative information related to aspirin in primary prevention does not seem to have adversely affected the prescription of aspirin for secondary prevention in these practices.

All practices involved in this study use clinical decision support software that reminds clinicians to consider aspirin in individuals with CVD. Until 2009 this system also prompted clinicians to con- sider aspirin in individuals with diabetes, chronic kidney disease (based on International Classification of Diseases, Ninth Revision, diagnoses or calculated creatinine clearance), or a calculated Framingham risk score $>10 \%$. The use of this software may have increased the recording of aspirin usage from 2007 to 2009 without any actual change in true underlying usage. From 2009 on these recommendations were turned off in some but not all 33 practices. $^{24}$

There are of course limitations to this analysis. Because aspirin is an over-the-counter medication, the use of EHR data to capture usage likely underestimates the percentage of the population actually using the medication. There is no reason to believe that the underestimated usage dramatically changed between study periods, in particular as it relates to

Figure 1. Percentage of all patients recorded as receiving aspirin therapy. AA, aortic aneurysm; CKD, chronic kidney disease; CVD, cardiovascular disease; DM, diabetes mellitus; HLD, hyperlipidemia; HTN, hypertension; PVD, peripheral vascular disease.

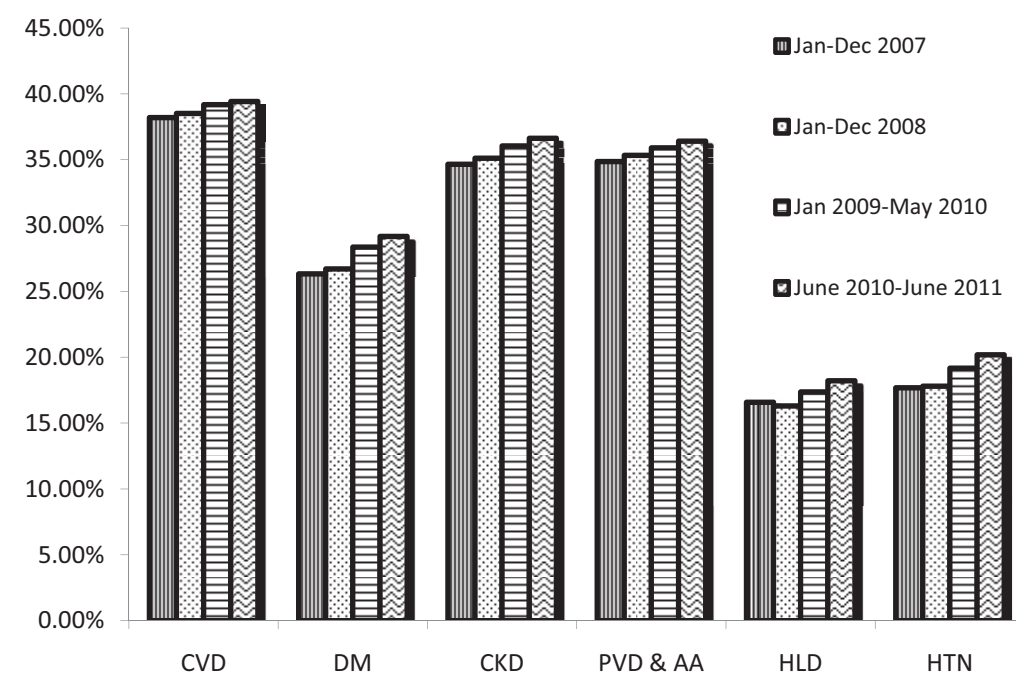


Figure 2. Percentage of patients with current cardiovascular disease (CVD) who are taking aspirin (ASA) versus patients with newly diagnosed CVD who were prescribed ASA within the first year of diagnosis.

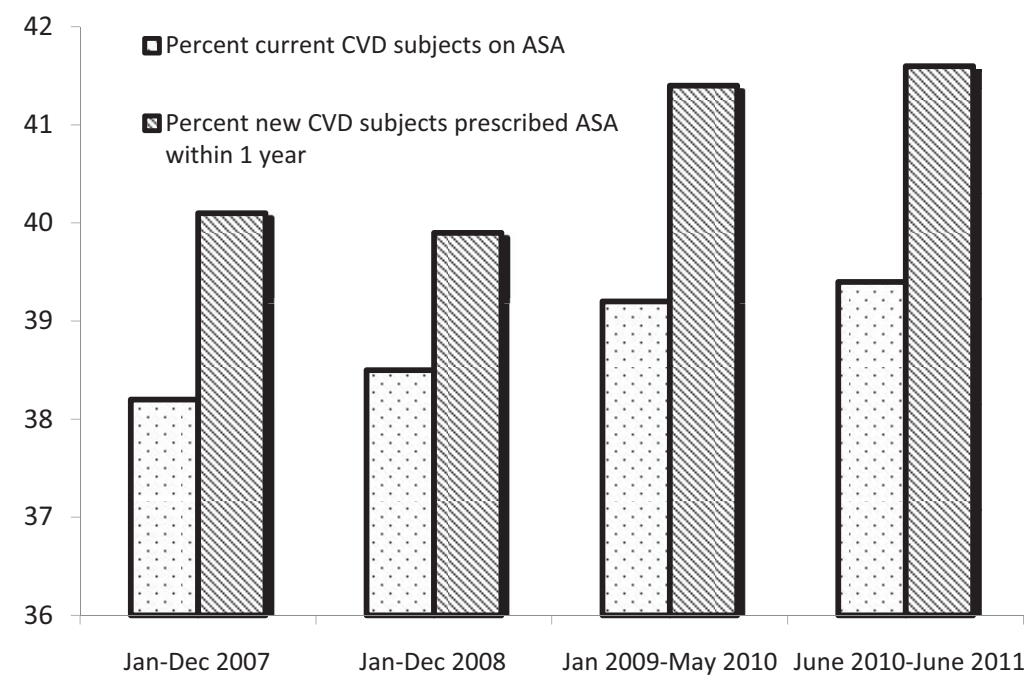

newly diagnosed individuals. This dataset comes from 11 distinct clinical organizations across 6 states that are not related except through their participation in eNQUIRENet; thus it is unlikely that widespread, independent medication reconciliation activities fully account for the results. The results for indi-

Figure 3. Percentage of all patients prescribed aspirin within 1 year of new diagnosis. Arrows indicate corresponding time of study publication or guideline change. AA, aortic aneurysm; ADA, American Diabetes Association; AHA, American Heart Association; ATTC, Antithrombotic Trialists' Collaboration; CKD, chronic kidney disease; CVD, cardiovascular disease; DM, diabetes mellitus; DOPPS, Dialysis Outcomes and Practice Patterns Study; HLD, hyperlipidemia; HTN, hypertension; JPAD, Japanese Primary Prevention of Atherosclerosis with Aspirin for Diabetes; POPADAD, Prevention of Progression of Arterial Disease and Diabetes Trial; PVD, peripheral vascular disease.

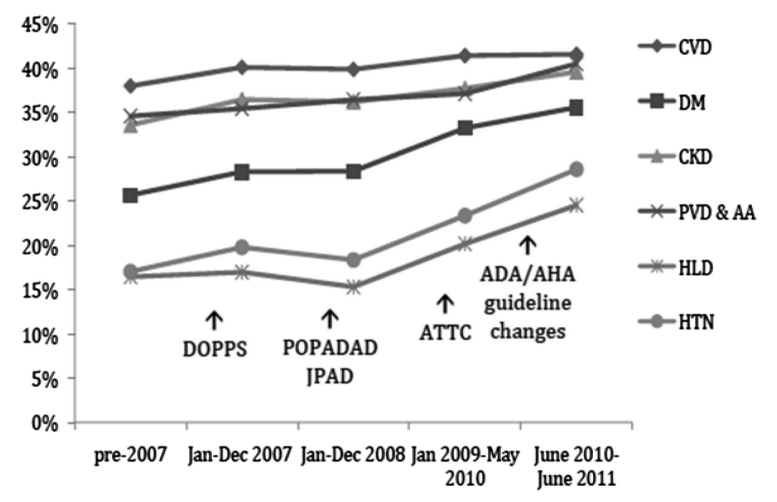

viduals who newly entered the cohorts across time periods also reduce the likelihood that medication reconciliation efforts over time could account for the findings. Thus the observed changes are not likely to be entirely due to recording artifact.

It is clear that many of the newly diagnosed patients placed on aspirin met criteria for primary prevention as outlined by the US Preventive Services Task Force ${ }^{2}$ or ADA/AHA guidelines ${ }^{16-18}$ at the time. Thus the clinicians in these practices cannot be criticized for following published guidelines, and some were subject to pay-for-performance evaluations based on increasing the use of aspirin for primary prevention. The inclusion of aspirin as a quality metric is likely to have blunted any impact of the new data from controlled trials or the subtle changes in the ADA/AHA guidelines. In addition, during this time period data were emerging that indicated low-dose aspirin may be beneficial in reducing risk of colon cancer. This could have blunted the impact of repeated negative studies for primary prevention of cardiovascular events, although meta-analysis supporting the use of lowdose aspirin for colon cancer prevention appeared long after the time period in question. ${ }^{25,26}$ Furthermore, the meta-analysis by Shesasai et $\mathrm{al}^{23}$ evaluated cancer mortality as well as CVD mortality and found no overall effect of aspirin use on cancer deaths.

With regard to aspirin prescriptions in the EHR, it is impossible to know the validity of the data at the 
population level. In some cases, the timing of a patient's diagnosis and initiation of aspirin therapy may not be captured accurately. It is possible to imagine patients who are well known to a practice, having been previously diagnosed with diabetes and prescribed aspirin, but the aspirin data are entered later, only after a prompt from the EHR. Likewise, a patient who was placed on aspirin in 2007 may have been discouraged from aspirin use at a later visit but refused to stop taking it. In any case, it is not likely that the reliability of the data changed enough over time to actually mask a decrease in the use of aspirin for primary prevention. The analysis of new diagnoses per time period would argue against this scenario; it is unlikely that new usage would go up over time if clinicians were actively discouraging aspirin use for primary prevention, and medication reconciliation activities would not be a major factor in individuals just entering the high risk cohorts. As aspirin can be obtained without a prescription and is generally considered to be harmless, it is more likely that patients did not report use and that true aspirin consumption is actually higher. While this is good news for the population with known CVD, it is potentially concerning for the primary prevention populations.

A strength of this analysis is that it includes multiple primary care organizations across multiple states using varying EHRs; thus it is unlikely that any organization-specific activity, such as medication reconciliation improvements, could explain the findings. Furthermore, the data are drawn from all individuals $>17$ years old in these practices and therefore do not represent sampling activities and as such do not require further statistical analysis to represent true changes in this population.

\section{Conclusions}

Translating research into practice is difficult. Stopping previously recommended treatments might be particularly difficult because both clinicians and patients must change previously held beliefs. It is known that there is a time lag between the release of recommendations and widespread clinical application $^{27}$; thus it is possible that aspirin use for primary prevention may slow over the next several years. Cancer prevention may create further pressure to increase the use of low-dose aspirin, but it is important to evaluate all-cause mortality in primary prevention studies to determine overall benefit, not just cancer-specific outcomes.
In theory, using aspirin for primary prevention in high-risk individuals makes sense: coronary artery disease exists well before an index event or before it is serendipitously found. Shiffman et $\mathrm{al}^{28}$ have proposed genetic testing to improve aspirin usage for primary prevention. Their model starts with the assumption that using aspirin for individuals with a $\geq 10 \%$ Framingham risk score for CVD is beneficial, a position not supported by the literature. Without new positive studies related to primary prevention it is difficult to understand ongoing support for aspirin usage in this context.

We thank Karl Hammermeister, MD, for providing access to the database used for this analysis and Elizabeth Staton for help with manuscript preparation. We also thank all the clinical organizations that participated in the original project and contributed data for use in this secondary analysis.

\section{References}

1. Hayden M, Pignone M, Phillips C, Mulrow C. Aspirin for the primary prevention of cardiovascular events: a summary of the evidence for the U.S. Preventive Services Task Force. Ann Intern Med 2002; 136:161-72.

2. Aspirin for the prevention of cardiovascular disease: U.S. Preventive Services Task Force recommendation statement. Ann Intern Med 2009;150:396-404.

3. Hennekens CH, Dyken ML, Fuster V. Aspirin as a therapeutic agent in cardiovascular disease: a statement for healthcare professionals from the American Heart Association. Circulation 1997;96:2751-3.

4. Peto R, Gray R, Collins R, et al. Randomised trial of prophylactic daily aspirin in British male doctors. $\mathrm{Br}$ Med J (Clin Res Ed) 1988;296:313-6.

5. Final report on the aspirin component of the ongoing Physicians' Health Study. Steering Committee of the Physicians' Health Study Research Group. N Engl J Med 1989;321:129-35.

6. Hansson L, Zanchetti A, Carruthers SG, et al. Effects of intensive blood-pressure lowering and lowdose aspirin in patients with hypertension: principal results of the Hypertension Optimal Treatment (HOT) randomised trial. HOT Study Group. Lancet 1998;351:1755-62.

7. Thrombosis prevention trial: randomised trial of low-intensity oral anticoagulation with warfarin and low-dose aspirin in the primary prevention of ischaemic heart disease in men at increased risk. The Medical Research Council's General Practice Research Framework. Lancet 1998;351:233-41.

8. Ridker PM, Cook NR, Lee IM, et al. A randomized trial of low-dose aspirin in the primary prevention of cardiovascular disease in women. $\mathrm{N}$ Engl J Med 2005;352:1293-304. 
9. De Berardis G, Sacco M, Strippoli GF, et al. Aspirin for primary prevention of cardiovascular events in people with diabetes: meta-analysis of randomised controlled trials. BMJ 2009;339:b4531.

10. Fleming T, Nissen SE, Borer JS, Armstrong PW. Report from the 100th Cardiovascular and Renal Drugs Advisory Committee meeting: US Food and Drug Administration: December 8-9, 2003 Gaithersburg, MD. Circulation 2004;109:e9004-5.

11. Ethier J, Bragg-Gresham JL, Piera L, et al. Aspirin prescription and outcomes in hemodialysis patients: the Dialysis Outcomes and Practice Patterns Study (DOPPS). Am J Kidney Dis 2007;50:602-11.

12. Belch J, MacCuish A, Campbell I, et al. The Prevention of Progression of Arterial Disease and Diabetes (POPADAD) trial: factorial randomised placebo controlled trial of aspirin and antioxidants in patients with diabetes and asymptomatic peripheral arterial disease. BMJ 2008;337:a1840.

13. Ogawa H, Nakayama M, Morimoto T, et al. Lowdose aspirin for primary prevention of atherosclerotic events in patients with type 2 diabetes: a randomized controlled trial. JAMA 2008;300:2134-41.

14. Baigent C, Blackwell L, Collins R, et al. Aspirin in the primary and secondary prevention of vascular disease: collaborative meta-analysis of individual participant data from randomised trials. Lancet 2009; 373:1849-60.

15. Fowkes FG, Price JF, Stewart MC, et al. Aspirin for prevention of cardiovascular events in a general population screened for a low ankle brachial index: a randomized controlled trial. JAMA 2010;303:841-8.

16. Goldstein LB, Adams R, Alberts MJ, et al. Primary prevention of ischemic stroke: a guideline from the American Heart Association/American Stroke Association Stroke Council: cosponsored by the Atherosclerotic Peripheral Vascular Disease Interdisciplinary Working Group; Cardiovascular Nursing Council; Clinical Cardiology Council; Nutrition, Physical Activity, and Metabolism Council; and the Quality of Care and Outcomes Research Interdisciplinary Working Group: the American Academy of Neurology affirms the value of this guideline. Stroke 2006;37:1583633.

17. Colwell JA. Aspirin therapy in diabetes. Diabetes Care 1997;20:1767-71.

18. Colwell JA. Aspirin therapy in diabetes. Diabetes Care 2004;27(Suppl 1):S72-3.
19. Pignone M, Alberts MJ, Colwell JA, et al. Aspirin for primary prevention of cardiovascular events in people with diabetes: a position statement of the American Diabetes Association, a scientific statement of the American Heart Association, and an expert consensus document of the American College of Cardiology Foundation. Diabetes Care 2010;33:1395402.

20. Hammermeister K. A cardiovascular risk reduction learning community in DARTNet. University of Colorado Denver: National Heart, Lung, and Blood Institute, Recovery Act Grant, NIH Challenge Grants and Partnerships Program. 2009.

21. D'Agostino RB Sr, Vasan RS, Pencina MJ, et al. General cardiovascular risk profile for use in primary care: the Framingham Heart Study. Circulation 2008;117:743-53.

22. Wolters Kluwer Health. Master Drug Data Base v2.5. Available from: http://www.nlm.nih.gov/research/ umls/sourcereleasedocs/current/MDDB. Accessed November 15, 2013.

23. Seshasai SRK, Wijesuriya S, Sivakumaran R, et al. Effect of aspirin on vascular and nonvascular outcomes: meta-analysis of randomized controlled trials. Arch Intern Med 2012;172:209-16.

24. Folks B, Leblanc WG, Staton EW, Pace WD. Reconsidering low-dose aspirin therapy for cardiovascular disease: a study protocol for physician and patient behavioral change. Implement Sci 2011;6:65.

25. Rothwell PM, Wilson M, Price JF, Belch JF, Meade TW, Mehta Z. Effect of daily aspirin on risk of cancer metastasis: a study of incident cancers during randomised controlled trials. Lancet 2012;379:1591601.

26. Rothwell PM, Price JF, Fowkes FG, et al. Shortterm effects of daily aspirin on cancer incidence, mortality, and non-vascular death: analysis of the time course of risks and benefits in 51 randomised controlled trials. Lancet 2012;379:1602-12.

27. Davis DA, Taylor-Vaisey A. Translating guidelines into practice. A systematic review of theoretic concepts, practical experience and research evidence in the adoption of clinical practice guidelines. CMAJ 1997;157:408-16.

28. Shiffman D, Slawsky K, Fusfeld L, Devlin JJ, Goss TF. Cost-effectiveness model of use of genetic testing as an aid in assessing the likely benefit of aspirin therapy for primary prevention of cardiovascular disease. Clin Ther. 2012;34:1387-94. 